\title{
On the relation between frequency estimates and recognition memory
}

\author{
GRANT HARRIS, IAN BEGG, and JOHN MITTERER \\ McMaster University, Hamilton, Ontario, L8S 4KI Canada
}

\begin{abstract}
The paper concerns the relation between frequency estimates and recognition decisions. Theories postulating that these two measures reflect independent retrieval processes and theories that postulate that frequency estimation and recognition are mutually dependent processes are discussed. Empirical results apparently supporting both positions are also reviewed. Results of experiments in which instructions and test methods are varied factorially and results of conditional and correlational analyses establishing a dependency between frequency estimates and recognition decisions are taken as evidence supporting the dependent process view. However, the results do not permit discrimination within this class of theories.
\end{abstract}

This paper concerns the relation between frequency estimation and recognition judgments. The position advocated in this article is that both tasks depend on access with the same memory information and that the same decision process subserves both tasks. To some extent the relation is forced, inasmuch as estimating that an item has occurred one or more times presupposes or includes a decision that the item has indeed occurred. However, this does not imply that recognition tests and frequency estimates, which are different tests of retention, are accomplished in the same manner. In fact, as we shall outline below, there are reasons to expect that different retrieval processes and different memory information are involved. First, however, let us consider the case for the dependency position, namely, that the same memory information accessed in the same way underlies performance in both tasks.

Several theories of frequency information are in agreement with the dependency position, in that they characterize frequency estimates as transformations of general information. For example, Hintzman and Block's (1971) multiple-trace theory assumes that each occurrence of an item establishes a separate trace. Recognizing an old item requires contact with a trace, and frequency judgments require the additional stage of estimating the number of such traces. Similarly, Anderson and Bower (1972) assume that each occurrence of an item establishes a list marker at the permanent address of the item. Recognition requires the presence of at least one list marker, and frequency judgments require estimation of the number of list markers. Tversky and Kahneman (1973) also postulate that frequency judgments do not depend on any frequency-specific information, but rather, they are direct inferences from the

This research was supported by NSERC (Canada) Grant A8122 to Ian Begg. Reprints can be obtained from lan Begg, Department of Psychology, McMaster University, Hamilton, Ontario L8S 4K1, Canada. John Mitterer is now at Trent University, Peterborough, Ontario. availability or ease of retrieving a memorial instance of the test event. Despite differences among them, these theories all characterize frequency estimates as subsequent to and dependent on recognition decisions.

There are also several lines of research evidence consistent with the dependency position. For example, variables that increase the accuracy of recognition judgments also increase the slope of the function relating judged frequency to actual frequency (e.g., Begg, 1974; Rowe, 1973; Underwood, Zimmerman, \& Freund, 1971). In addition, the slope of the frequency function is about the same whether subjects are specifically instructed to attend to frequency during study or are simply told to study the items for a later memory test (Flexser \& Bower, 1975; Howell, 1973; see also Hasher \& Chromiak, 1977). Further, there is a direct relation between frequency and memory, in that words given relatively high frequency estimates are relatively likely to be retained on a later test (Begg, 1974; Underwood et al. 1971).

Although there is some convergence of theory and data with regard to the dependency position, there are also divergent points of view and some discrepant findings. Underwood (1969) assumes that the memory trace for an item is a bundle of attributes, one of which is specifically sensitive to event frequency. The frequency attribute subserves frequency estimation, while other attributes subserve other memory tasks. Although Underwood does assume an attribute specific to frequency, he also assumes that recognition decisions are accomplished exclusively by reference to the frequency attribute (Underwood et al., 1971), so that attribute theory is not basically at odds with the dependency position. In contrast, Proctor's (1977) strength-eventstore account posits both different sources of information and different demands of frequency and recognition tests. By the account, there are two separate sources of information about an event, each serving a different function. First, there is a general reserve of memory 
strength used for judgments like recognition and recency. Second, there is a more specific episodic store that provides information about the context in which the event occurred, used for estimates of frequency. Since these sources of information are memorially separate, there is no reason to expect dependency between the two measures.

Proctor (1977) presents evidence consistent with his account and problematic for dependency positions. Frequency estimates can be scored to make them comparable to recognition decisions, by defining "old" as any frequency estimate greater than zero (see Begg \& Rowe, 1972). Using such a procedure, Proctor found that the frequency test yielded a higher hit rate than the recognition test (.84 vs. .76 ), but with no reliable difference in false alarms (.21 vs. .19). The finding that old items are more discriminable from new ones on a frequency test than in recognition directly is certainly troublesome for the dependency position. However, the finding may not be general. Malmi (1977) presents data in which frequency estimation gives a higher hit rate than recognition testing (.85 vs. .73 ), but with a higher false alarm rate as well ( .40 vs. .20 ), suggesting that subjects adopt a more lenient criterion for classifying items as old in the frequency test. Underwood (1972) contrasted a multiple-choice test in which subjects either recognized old items or chose the most frequent item from each set of alternatives. The two procedures yielded equivalent error rates, and the errors tended to be the same items for both groups $(r=.74)$. Both experiments in the present paper directly compare recognition accuracy in discriminating old items from new ones directly on a recognition test and as inferred from frequency estimates. The objective is simply to assess the generality of Proctor's finding.

Another problem for the dependency position arises from recent approaches to human memory that emphasize encoding specificity (e.g., Watkins \& Tulving, 1975 ) and transfer-appropriate processing (e.g., Morris, Bransford, \& Franks, 1977). By such accounts, encoding is both relatively flexible and focused toward the expected manner of test. Consequently, optimal performance on a particular retention test requires the prior encoding of information appropriate for that test. Since frequency estimation and recognition tests are different tests, we could expect an interaction between study and test, with appropriately instructed subjects performing better than inappropriately instructed subjects. In support of such accounts, Howell (1973) found that recall was better after memory instructions than after frequency instructions, while frequency estimates were unaffected by the instructions. However, there are as yet no studies that specifically examine performance on recognition and frequency tests after recognition and frequency instructions. Since there is no guarantee that results from recall also characterize recognition, both experiments in the present series orthogonally varied study instructions and test in order to examine the question more directly.

In summary, although the view that frequency estimates and recognition judgments require similar processing of a common memory substrate has considerable support, there are some cogent reasons for reexamining the relation. The primary interest of the present experiments is with two questions. First, does frequency estimation allow more accurate discrimination of old from new items than does recognition testing? Second, do instructions to study items for frequency or recognition make any difference on later tests of frequency or recognition? By the dependency position, the answer should be no in both cases.

\section{EXPERIMENT 1}

In the first experiment, frequency estimation and recognition memory were assessed for words presented one, two, or three times, relative to new items. Subjects received either a recognition or a frequency test following a common study list. Prior to study, half the subjects were instructed that there would be a later frequency test and half were told to expect a later test of recognition. Following study, half the subjects in each instruction condition were given a frequency test and half, a recognition test.

Based on previous research, the instructions should not affect frequency estimates. Accordingly, if recognition involves the same process of discrimination among items, based upon the same memory information, as does frequency estimation, recognition should not be affected by the instructions either. Second, it is also of interest to determine whether the two methods of test give evidence of differential discriminability of remembered items, as found by Proctor (1977).

In the experiment, subjects tested by recognition were then tested by frequency, and vice versa. The point of the second test was to examine performance in the second test conditionalized on performance in the first test. The virtue of such an analysis is that the dependency position predicts that the hypothesis of independent performance will be rejected, a more satisfactory state of affairs than the acceptance of the null hypothesis with regard to instructions. Several recent papers (e.g., Bacon, 1979; Begg, 1979; Flexser \& Tulving, 1978) have based decisions on the outcome of such conditional analyses, which are sensitive enough to support conclusions that processes are independent in some cases, dependent in others. An inevitable shortcoming of repeated testing is that the influence of the first test on the second is unknown. However, in the cases cited above, effects of prior testing were not substantial, judged by control conditions. Accordingly, we employed the procedure, and we interpret the results in combination with the results of other analyses. The expectation is, obviously, that recognition and frequency tests will show substantial dependency over the test occasions. 


\section{Method}

Subjects. Forty introductory psychology students served as subjects and were paid $\$ 2$ or received course credit. There were 10 subjects in each of four conditions.

Materials and Procedure. The 120 critical items were chosen from the Paivio, Yuille, and Madigan (1968) norms such that Thorndike-Lorge frequency exceeded 20 and rated imagery was less than 4.10. Twelve additional items were chosen to act as fillers. Of the 120 critical nouns, 30 were chosen at random to occur once in the presentation list, 30 were chosen to occur twice, 30 to occur three times, and 30 not to occur in the presentation list. Repetitions of critical items and fillers required a 204-item presentation list. Twelve fillers (and repetitions) occurred at the beginning and 12 occurred at the end of the list. Critical items that were repeated occurred at a spacing of from one to seven intervening items (mean $=4.5$ ). The entire list was tape-recorded at a rate of approximately one item every $2.5 \mathrm{sec}$. Subjects in two conditions received recognition study instructions and were told that afterward they would receive a recognition test and they should try to remember the words as they occurred. The other two groups received the frequency study instructions, which were identical except that the subjects were told to expect that they would be asked how many times each word occurred and to remember the number of occurrences for each word.

Every subject then listened to the same tape-recorded list. Afterward, each subject was presented the same final test list, composed of the 120 randomly ordered critical items. One of the two recognition study groups and one of the frequency study groups were instructed to indicate beside each item whether or not it occurred in the list by writing "yes" or "no." The other two groups were instructed to indicate the number of times each item had occurred in the study list (zero, one, two, or three). After completing the first test, the test sheets were collected, and each subject then completed the other task, using a new response sheet. The entire procedure required about $30 \mathrm{~min}$. Subjects were tested individually or in small groups.

\section{Results and Discussion}

Throughout the paper, means are accompanied by standard deviations in parentheses, and $\alpha$ was set at .05 . For clarity of exposition, results are reported separately for each measure of interest.

Instructions. Instructions were associated with no reliable main effects or interactions in analyses of marginal or conditional measures of recognition or frequency. For example, on the frequency-estimation test immediately following study, estimates increased over true frequencies from 0 to 3 , with means of .63, 1.17, 1.59 , and 1.90 following recognition instructions and $.65,1.24,1.69$, and 1.88 following frequency instructions. Similarly, for measures of recognition derived from frequency estimates and for recognition judgments themselves, the difference between hits and false alarms ranged from .35 to .37 over the four combinations of study instructions and manner of test. Accordingly, along with Flexser and Bower (1975) and Howell (1973), we failed to find instructional effects in frequency estimation, and we generalized that failure to recognition tests as well. Although the null hypothesis cannot be accepted, it is possible to conclude that any effects the instructions may have had are too small to be interesting. The remaining analyses thus ignore instructions, although the question will be addressed again in Experiment 2 .
Recognition memory. In this section the dependent measure of interest is the proportion of test items classified as old. "Oldness" was defined by a response of "yes" on the recognition tests; thus, for items presented one, two, or three times, the measure defines hits, and for items presented zero times, the measure defines false alarms. For the frequency tests, oldness was defined by a numerical response of one, two, or three, again measuring hits for actually presented items and false alarms for new ones. In each analysis to follow, there was a large and reliable effect of frequency, simply meaning that recognition benefits from extra study occasions. Of more interest are effects of the other variables both alone and in interaction with frequency.

The first analysis concerned the effects of type of test on recognition. Means are presented in the top two rows of Table 1. It is clear that the hit rate was higher on the frequency test than on the recognition test by .11 to .13. However, the false alarm rate was .11 higher as well, so that the difference between test types was simply a main effect $[F(1,36)=8.71]$. The results do replicate Proctor's (1977) finding that frequency testing exceeds recognition testing in hit rate, but the difference in hit rates was paralleled by an equal difference in false alarms (cf. Malmi, 1977). That is, the difference between tests reflects a difference in criterion bias, not a difference in the discriminability of old items from new ones. The question will be addressed again in Experiment 2; however, at this point, we can conclude that the finding reported by Proctor does not generalize to the present case, perhaps because of procedural differences or perhaps because the original result was of small magnitude.

The second analysis briefly examines recognition measured on the second test, in comparison with recognition on the first test. First, consider the later frequency test that followed the immediate recognition test whose means appear in the second row of Table 1. The propor-

Table 1

Memory Performance as a Function of Presentation Frequency and the Manner of Test

\begin{tabular}{|c|c|c|c|c|c|c|c|c|}
\hline \multirow[b]{3}{*}{ Test } & \multicolumn{8}{|c|}{ Actual Frequency } \\
\hline & \multicolumn{2}{|c|}{0} & \multicolumn{2}{|c|}{1} & \multicolumn{2}{|c|}{2} & \multicolumn{2}{|c|}{3} \\
\hline & Mean & $\mathrm{SD}$ & Mean & SD & Mean & $\mathrm{SD}$ & Mean & SD \\
\hline & \multicolumn{8}{|c|}{ Experiment 1} \\
\hline Frequency & .41 & .20 & .69 & .15 & .82 & .11 & .84 & .11 \\
\hline \multirow[t]{2}{*}{ Recognition } & .30 & .19 & .57 & .14 & .69 & .15 & .73 & .18 \\
\hline & \multicolumn{8}{|c|}{ Experiment 2} \\
\hline Frequency & .24 & .18 & .70 & .14 & .85 & .12 & .92 & .08 \\
\hline Recognition & .16 & .13 & .61 & .24 & .75 & .23 & .85 & .17 \\
\hline
\end{tabular}

Note-Entries are proportions of items called "old" on the recognition tests and items judged to have occurred one, two, or three times on the frequency tests. Thus the entries are false alarms for items actually presented zero times and hits otherwise. 
tion of items classified as old increased less steeply from new to old items on the second test than on the first, resulting in an interaction between presentation frequency and time of test $[F(3,54)=11.8]$; means on the second test for items presented zero, one, two, and three times were $.45(.22), .61(.19), .64(.20)$, and .71 (.17). Similarly, the immediate frequency test, whose means appear in the first row of Table 1, was followed by a recognition test; again, the means increased less steeply from new to old items on the second test $[F(3,54)=4.67]$, with respective values of $.47(.18)$, $.66(.15), .80(.14)$, and $.79(.12)$. Thus, in each case, comparison of the second test with the first simply shows decreased discriminability of old items from new ones, regardless of changes in type of test.

The final analysis concerns the dependency or independence of recognition judgments on frequency estimates. The first test of dependency was to conditionalize recognition responses on the second test upon the frequency judgments for the same items on the first test. The proportions of items called old, given initial frequency estimates of zero, one, two, or three, were $.31(.10), .74(.16), .83(.13)$, and $.94(.09)$, respectively, showing a considerably steeper slope over judged frequency than in the previous analysis over actual frequency. The second test of dependency was to calculate tetrachoric correlations (Davidoff \& Goheen, 1953) between the two tests. For items presented zero times, .59 were called new on the first test, .54 were called new on the second, with .43 called new on both (yielding $r_{t}=.68$ ). Similarly, for items presented one time, .31 were called new on the first test, .35 on the second, and .21 on both $\left(r_{t}=.71\right)$. For items presented two times, the respective means were $.18, .21$, and .12 $\left(r_{t}=.80\right)$, and for items presented three times, the means were $.16, .21$, and $.11\left(\mathrm{r}_{\mathrm{t}}=.80\right)$. Thus correlations between tests were substantial, indicating that items tended to be treated in the same manner on both tests. Consequently, recognition judgments are not independent of frequency estimates.

Frequency estimates. Mean frequency estimates for items presented zero, one, two, or three times on the immediate frequency test and on the second frequency test that followed recognition are presented in Table 2. It is clear that the slope of the function relating true to judged frequency is steeper on the first test than on the second $[F(3,108)=6.84]$, indicating a loss of the ability to discriminate items on the basis of frequency over occasions, as was the case with recognition judgments. Note that the tendency to overestimate less frequent items and to underestimate more frequent items increases over test occasions, as previously reported by Begg (1974).

The final analysis concerns dependency of frequency estimates on prior recognition. Table 2 presents mean frequency estimates for items called old or new on the prior test. It is clear that frequency estimates were low and invariant for items called new, but higher and with some increase over true frequency for items called old, yielding a reliable interaction $[F(3,54)=8.61]$. Thus recognition, including false recognition, is necessary for frequency estimates to increase with true frequency. Therefore, frequency estimates are not independent of recognition judgments.

\section{Summary and Conclusions}

Subjects were instructed that they would later be tested for recognition or frequency, then were later tested by either test. Instructions had no reliable or interesting effects on any measure of performance. However, the two tests did differ in the proportion of items classified as old, with the frequency test higher than the recognition test by .11 in false alarms and by .12 in hits. Thus the frequency test in some way lowers the criterion for accepting a test item as old, but it does not make old items any more discriminable from new ones than does the recognition test. However, old items were less discriminable from new ones on the second test than on the first, regardless of the nature of either test or the measure employed. It was also found that the two tests were not independent. Thus the proportion of items classified as old on the delayed recognition test

Table 2

Frequency Estimates in Several Conditions

\begin{tabular}{|c|c|c|c|c|c|c|c|c|}
\hline & \multicolumn{8}{|c|}{ Actual Frequency } \\
\hline & \multicolumn{2}{|c|}{0} & \multicolumn{2}{|c|}{1} & \multicolumn{2}{|c|}{2} & \multicolumn{2}{|c|}{3} \\
\hline & Mean & SD & Mean & SD & Mean & SD & Mean & SD \\
\hline & \multicolumn{8}{|c|}{ Experiment 1} \\
\hline $\begin{array}{l}\text { First Frequency Test } \\
\text { Second Frequency Test }\end{array}$ & .64 & .37 & 1.21 & .43 & 1.64 & .39 & 1.89 & .43 \\
\hline All Items & .72 & .35 & 1.04 & .34 & 1.30 & .49 & 1.49 & .46 \\
\hline Items Called "Old" & 1.29 & .40 & 1.61 & .39 & 1.53 & .46 & 1.84 & .37 \\
\hline \multirow[t]{2}{*}{ Items Called "New" } & .53 & .33 & .57 & .36 & .52 & .32 & .56 & .39 \\
\hline & \multicolumn{8}{|c|}{ Experiment 2} \\
\hline Running Task & & & 1.22 & .18 & 2.15 & .25 & 2.64 & .46 \\
\hline Delayed Task & .33 & .25 & 1.00 & .36 & 1.55 & .41 & 1.97 & -44 \\
\hline
\end{tabular}


increased much more steeply over judged than true frequency, and items tended to be classified in the same recognition category on both tests. Similarly, frequency estimates on the delayed test were much higher for items judged to be old on the immediate test than for items judged to be new; in fact, the latter judgments showed no change over true frequency. Overall, the results are consistent with the view that frequency estimation includes a recognition process whereby test items are first assessed as new or old prior to numerical estimation.

\section{EXPERIMENT 2}

Because the procedure of the first experiment does not guarantee that subjects obeyed the instructions to attend to frequency, the second experiment involved a stronger instructional manipulation. Half the subjects judged the frequency of each item as the list was presented, and the others produced running recognition judgments, thus guaranteeing that the items were indeed studied in the requested manner. Following study, subjects were tested either by recognition or frequency, again allowing a test of whether studying items for frequency or recognition has any effect on a later frequency or recognition task. In neither case was the instructional difference expected by the theories that assume that repetition has a common effect upon the memory information that mediates both tasks. Again, in this experiment it was possible that the final test could show more discriminability of old items from new ones in frequency estimation than in recognition. That is, we can again assess the generality of Proctor's (1977) finding.

\section{Method}

Subjects. Forty McMaster University students served as subjects and were paid $\$ 2$ or received course credit for their participation.

Materials and Procedure. One hundred nouns were selected from the Paivio et al. (1968) norms. For each one, ThorndikeLorge frequency was greater than 10 and I was less than 4.10. These 100 words were critical items for the experiment. Another 75 nouns were chosen (frequency $>10, I<4.60$ ) to act as fillers. From the set of 100 critical items, 75 were chosen at random to occur in the presentation list. Of these, 25 were randomly chosen to occur once, 25 to occur twice, and 25 three times. The presentation list was composed of 150 different words (75 critical and 75 fillers), and the repetitions of critical and filler items required a 290 -item presentation list. A series of fillers composed the first part of the study list, followed by the critical items, followed again by fillers. All items that were repeated occurred at a spacing of between 10 and 50 intervening items $($ mean $=31.1)$.

The entire 290-item list was tape-recorded at a rate of one item every $5 \mathrm{sec}$. Subjects were randomly divided into four groups. Two of the groups (recognition) were informed that they would hear a list of words, some of which were repeated up to three times, and these subjects were then instructed to indicate on a response sheet, for each word, whether or not they had heard that word before in the list. The other two groups (frequency) received the same instructions, except that they were instructed to indicate the number of previous occurrences for each word they heard. All subjects then listened to the same tape-recorded list and afterward were given a common final test sheet containing the same randomly ordered 100 critical items. One of the two recognition study groups and one of the two frequency study groups were instructed to indicate beside each of the critical items whether or not the word had occurred in the presentation list. The other two groups were instructed to indicate how many times each of the words on the final test list had occurred in the list. The entire procedure took about $45 \mathrm{~min}$, with subjects tested individually or in small groups.

\section{Results and Discussion}

There are two preliminary points. First, despite instructions, some subjects produced frequency estimates greater than three; such responses, with a mean of 4.9 , constituted $3.5 \%$ of all responses on the frequency tests and were scored as three in all analyses. Second, 3 of the 20 subjects in the running recognition test produced unscorable response sheets by omitting one item; these subjects were ignored in analysis of the continuous tasks, but not, of course, in the final tests.

Instructions. At this point, we are tempted to accept the null hypothesis, since again there was no reliable effect of instructions on any measure on the later tests. For example, over the eight comparisons between instructions on the delayed recognition of items with various presentation frequencies, the difference between instructions ranged from .09 to -.10 haphazardly, with a mean of, 00 . However, to be safe, let us simply conclude that instructions, despite the very strong manipulation, failed to produce any reliable effects. Although we do not conclude that instructions had no effect, we do conclude that any effect they may have had is singularly hard to detect and is of minimal interest.

Recognition memory. On the continuous test, performance was extremely accurate. The likelihoods that items were classified as old on their first, second, and third appearances were $.18(.15), .91(.09)$, and .97 $(.10)$ on the frequency test and $.12(.08), .88(.15)$, and $.96(.07)$ for the recognition test. There is obviously no evidence that frequency testing shows better discriminability than recognition testing on continuous tests.

The mean proportions of items classified as old on the test following presentation are presented in the bottom half of Table 1 . The frequency test was between .07 and .10 higher in hit rates than was the recognition test, but there was also a difference of .08 in false alarms. Therefore, the difference was simply a main effect $[F(1,36)=4.28]$, in agreement with Experiment 1 but not with Proctor (1977). In fact, the results were almost numerically identical to Proctor's except for the difference in false alarms found here. We thus agree with Malmi (1977) that the frequency test procedure results in a lowered criterion for accepting items as old, but with no concomitant change in the discriminability of old items from new ones.

Frequency estimates. Mean estimates for items on their first, second, and third appearances on the continuous test and mean estimates for items with true fre- 
quencies of zero, one, two, and three on the final test are presented in Table 2 . Across the old items, incrementation was steeper on the continuous test than on the delayed test $[F(2,18)=12.6]$, as found by Begg (1974). Again, discriminability attenuated with delay.

\section{GENERAL DISCUSSION}

The research reported in this paper supports a theoretical position that recognition and frequency estimation are dependent retention measures because they involve the same basic memory information. The consistent lack of a differential effect of instructions and orienting task, for either frequency or recognition, leads naturally to the conclusion that the effects of repetition upon memory are the same regardless of instructions. That is, recognition instructions lead to an encoding strategy that is just as appropriate for frequency estimation as is the encoding strategy produced by frequency instructions. The same result obtains for the effects of frequency and recognition instructions upon performance on a recognition test.

Furthermore, results reported here and those reviewed in the introduction establish that both kinds of test are influenced by the same variables and yield the same levels of item discrimination. Manipulations that influence discriminability in recognition judgments also affect the slope of the function relating judged to true frequency. Although the two kinds of test may yield differences in a subject's criterion for saying "old," there is evidence that they do not yield different levels of item discrimination. Thus, Proctor's (1977) result is not replicated; that finding is of limited generality, as well as small magnitude.

Finally, conditional analyses establish a dependency between frequency estimates and the outcome of earlier recognition decisions and between recognition judgments and earlier frequency estimates. All the results are consistent with theoretical positions that assert that some kind of item discrimination is a necessary component to both tasks and that both tasks are mediated by the same memory information. A strong conclusion of this paper is that there is no necessity to postulate different encoding strategies for recognition and frequency tasks, nor is there any necessity to postulate that the two tasks are accomplished with reference to different memory information. We conclude that frequency estimates and recognition decisions are highly dependent measures of memory retention because they involve the same information used in pretty much the same way.

There exists some disagreement, however, about how this common memory information is to be conceptualized and about how it is used in making frequency and recognition judgments. Theories differ in the way that they hypothesize that repetitions affect memory. The research reported here does not address the question of whether frequency information is explicitly represented or inferred from more general memory information, for example. Such theoretical difference may only exist at a superficial verbal level, and the theories may not make differential predictions. Later developments in research and theory, particularly in more complex situations, may permit some determination.

\section{REFERENCES}

Anderson, J. R., \& Bower, G. H. Recognition and retrieval processes in free recall. Psychological Review, 1972, 79, 97-123.

Bacon, F. T. Credibility of repeated statements: Memory for trivia. Journal of Experimental Psychology: Human Learning and Memory, 1979, 5, 241-252.

BEGG, I. Estimation of word frequency in continuous and discrete tasks. Journal of Experimental Psychology, 1974, 102, 1046-1052.

BEGG, I. Trace loss and recognition failure of unrecalled words. Memory \& Cognition, 1979, 7, 113-123.

BegG, I., \& Rowe, E. T. Continuous judgments of word frequency and familiarity. Journal of Experimental Psychology, $1972,95,48-54$.

Davidoff, M. D., \& GoheEN, H. W. A table for the rapid determination of the tetrachoric correlation coefficient. Psychometrika, 1953, 18, 115-121.

Flexser, A. J., \& Bower, G. H. Further evidence regarding instructional effects on frequency judgments. Bulletin of the Psychonomic Society, 1975, 6, 321-324.

Flexser, A. J., \& Tulving, E. Retrieval independence in recognition and recall. Psychological Review, 1978, 85, 153-171.

Hasher, L., \& Chromiak, W. The processing of frequency information: An automatic mechanism? Journal of Verbal Learning and Verbal Behavior, 1977, 16, 173-184.

Hintzman, D. L., \& Block, R. A. Repetition and memory: Evidence for a multiple trace hypothesis. Journal of Experimental Psychology, 1971, 88, 297-306.

Howell, W. C. Storage of events and event frequencies. Journal of Experimental Psychology, 1973, 98, 260-263.

Malmi, R. A. Context effects in recognition memory: The frequency attribute. Memory \& Cognition, 1977, 5, 123-130.

Morris, C. D., Bransford, J. D., \& Franks, J. J. Levels of processing versus transfer appropriate processing. Journal of Verbal Learning and Verbal Behavior, 1977, 16, 519-534.

Paivio, A., Yuille, J. C., \& Madigan, S. Concreteness, imagery and meaningfulness values for 925 nouns. Journal of Experimental Psychology Monograph Supplement, 1968, 76(1, Pt. 2).

Proctor, R. W. The relationship of frequency judgments to recognition. Journal of Experimental Psychology: Human Learning and Memory, 1977, 3, 679-689.

Rowe, E. J. Frequency judgments and recognition of homonyms. Journal of Verbal Learning and Verbal Behavior, 1973, 12, 440-447.

TVersky, A., \& Kahneman, D. Availability: A heuristic for judging frequency and probability. Cognitive Psychology, 1973, $5,207-232$.

Underwood, B. J. Attributes of memory. Psychological Review, $1969,76,559-573$.

UNDERWOOD, B. J. Word recognition, memory and frequency information. Journal of Experimental Psychology, 1972, 94, 276-283.

Underwood, B. J., Zimmerman, J., \& Freund, J. S. Retention of frequency information with observations on recognition and recall. Journal of Experimental Psychology, 1971, 87, 149-162.

Watkins, M. J., \& Tulving, E. Episodic memory: When recognition fails. Journal of Experimental Psychology: General, $1975,104,5-29$. 